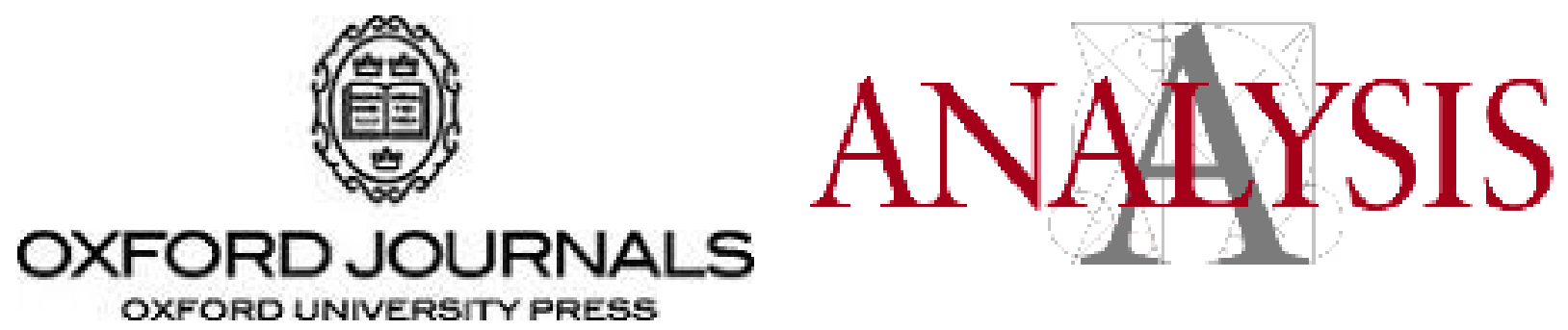

Proxy Functions and Inscrutability of Reference

Author(s): Steven L. Reynolds

Reviewed work(s):

Source: Analysis, Vol. 54, No. 4 (Oct., 1994), pp. 228-235

Published by: Oxford University Press on behalf of The Analysis Committee

Stable URL: http://www.jstor.org/stable/3328810

Accessed: 28/05/2012 16:09

Your use of the JSTOR archive indicates your acceptance of the Terms \& Conditions of Use, available at http://www.jstor.org/page/info/about/policies/terms.jsp

JSTOR is a not-for-profit service that helps scholars, researchers, and students discover, use, and build upon a wide range of content in a trusted digital archive. We use information technology and tools to increase productivity and facilitate new forms of scholarship. For more information about JSTOR, please contact support@jstor.org. 


\section{Proxy Functions and Inscrutability of Reference}

\section{STEVEN L. ReYNOLDS}

Quine's favorite argument for the inscrutability of reference relies on reinterpretation using proxy functions:

A proxy function is any explicit one-to-one transformation, $f$, defined over the objects in our purported universe. By 'explicit' I mean that for any object $x$, specified in an acceptable notation, we can specify $f x$. Suppose now we shift our ontology by reinterpreting each of our predicates as true rather of the correlates $f x$ of the objects $x$ that it had been true of. Thus, where ' $P x$ ' originally meant that $x$ was a $P$, we reinterpret ' $P x$ ' as meaning that $x$ is $f$ of a $P$. Correspondingly for two-place predicates and higher ... We leave all the sentences as they were, letter for letter, merely reinterpreting. The observation sentences remain associated with the same sensory stimulations as before, and the logical interconnections remain intact. Yet the objects of the theory have been supplanted as drastically as you please. ([12], pp. 31-2)

The penultimate sentence rehearses Quine's account of the evidence for translation: it consists of sensory stimulations that would prompt utterance, or assent or dissent, and also observable relations to other sentences of the native language. Presumably the idea of the argument is that any evidence for reference must also be evidence for translation. If two interpretations are supported equally by all possible evidence for translation, they must also be supported equally by all possible evidence for reference. So if the proxy function and standard interpretations cannot be distinguished by the evidence for translation, reference is inscrutable.

Quine tells us explicitly how to assign referents to subsentential expressions, but not how to find the corresponding sentence translations. Much of the literature on the proxy function argument assumes however that assignments of referents to subsentential expressions yield translations of the containing sentences by way of a Davidsonian theory of truth/meaning (Wallace [2], Field [3], Davidson [1]). Quine endorses the project of producing such a theory, ${ }^{1}$ so it seems likely that he has it in mind for this purpose. The assignments of referents to expressions are axioms of the theory, and, together with the other axioms (syntax and

1 '... it was left to Davidson to recognize Tarski's theory of truth as the very structure of a theory of meaning. This insight was a major advance in semantics.' (Quine [10] p. 38.) 
projection rules), they allow the derivation of truth conditions for each sentence. The object language sentence is translated in the statement of its truth condition, and the theory is empirically supported to the extent that the translations thus produced are empirically acceptable. (If the meta-language includes the object language, empirically acceptable translations may be guaranteed by designing the theory to generate homophonic truth conditions.)

A simple example will be useful later. Suppose we consider the sentence 'London is foggy'. On the standard reference scheme 'London' refers to London, and 'is foggy' is true of $x$ if and only if $x$ is foggy. 'London' is a name, and 'is foggy' is a predicate. Suppose we also have the projection rule 'A sentence of the form $F a$, where $F$ is a predicate true of all and only G's, and $a$ is a name that refers to $b$, is true if and only if Gb'. We can then derive " $L o n d o n$ is foggy" is true if and only if London is foggy'. 'London is foggy' (now in the meta-language) is thus the standard translation of the object language sentence 'London is foggy'.

Let $f$ represent a proxy function that maps London to Mt. Everest, Mt. Everest to the moon, the moon to London, and everything else to itself (Kirk [5], p. 116). According to the new reference scheme 'London' refers to $f$ (London) [i.e. Mt. Everest], and 'is foggy' is true of $x$ if and only if the $f$-inverse of $x$ is foggy. Using the above projection rule we can then derive " "London is foggy" is true if and only if $f$ (London) is such that its $f$-inverse is foggy'. So the proxy function altered translation is ' $f$ (London) is such that its $f$-inverse is foggy'.

We obtain this translation if we change the reference scheme, as Quine requires, leaving the rest of the theory untouched. But there is evidence in Pursuit of Truth that Quine has in mind a different proxy function reinterpretation. He says '.. divergent interpretations of the words in a sentence can so offset one another as to sustain an identical translation of the sentence as a whole. It is what I have called inscrutability of reference ...' ([12], p. 50). The above proxy function translation is obviously not an identical translation.

John Wallace suggests obtaining identical translations by adding to the theory of truth/meaning an axiom characterizing the proxy function, thus allowing derivation of either homophonic (if the metalanguage contains the object language), or standard, truth conditions ([13], p. 155). I suppose Quine must have Wallace's amendment in mind, since there seems to be no other way to get homophonic (or standard) translations starting from a proxy function altered reference scheme. (If, in addition to changing the reference scheme, we were to change the projection rules or syntax of the theory to permit the desired deductions, it would be doubtful that the result indicated inscrutability of reference.) 
But adding an axiom to a theory does not prevent any previously available deductions. So a Wallace-style reinterpretation yields two different statements of truth conditions for each sentence. For 'London is foggy' it yields as translations both 'London is foggy' and ' $f$ (London) is such that its $f$-inverse is foggy'. This seems a disadvantage, but perhaps we could still regard it as empirically equivalent to the standard interpretation if the two translations were equally supported by the evidence for translation.

So, although Quine intends his reinterpretation to result in homophonic translations, it will be empirically equivalent to the standard interpretation only if 'London is foggy' may be equally well translated by 'London is foggy' and by ' $f($ London) is such that its $f$-inverse is foggy'. Are these equally good translations, on Quine's own account of the evidence for translation?

In radical translation of a new language, or in learning one's native language, according to Quine, the evidence consists of environmental effects on the utterances of those who already speak it: sensory stimulations of the speaker and the resulting utterances and assents or dissents.

To learn a language is to learn to utter and accept its sentences under stimuli relevantly similar to those under which other speakers would utter or accept them. Good translation, on the other hand, matches sentences of the target language to sentences of a previously mastered language in a way that sufficiently preserves relevant stimulus-to-utterance patterns. At first one matches native sentences one has heard to sentences of one's own language that would be produced, assented to and dissented from in similar circumstances, and then, gradually, one constructs a systematic way of finding sentences to match even those sentences of the native language one hasn't heard.

The most important pieces of evidence, because most copiously available, are the stimulations that prompt assent to or dissent from a sentence. There has been a shift in Quine's characterization of this evidence. In Word and Object, observation sentences were to be translated, where possible, by matching them to sentences of similar stimulus meaning ([8], pp. 3235 ). The stimulus meaning of a sentence consists of those activations of sensory receptors that would alter tendencies to assent to or dissent from the sentence. It seems however that translation should not be impeded by differences in the configuration of the sensory receptors, which makes it hard to characterize the relevant similarity of stimulus meaning.

In response to this difficulty, in Pursuit of Truth, Quine claims instead that the translator should first seek sentences that would be assented to or dissented from in relevantly similar perceptual situations ([12], pp. 40-44). We are able to recognize similarity of perceptual situations, he says, 
although we may often be unable to indicate precisely the relevant shared features.

This characterization of the evidence for translation relaxes Quine's individualism. A perceptual situation, unlike a stimulus meaning, may include features of the environment beyond the subject's sensory receptors, such as the causes of their activation. I shall not discuss the familiar causal theory of reference objection to the proxy function argument however (Devitt [2], pp. 188-91; Kirk [5], pp. 116-27). Instead I shall raise an objection that applies to both of Quine's accounts of the evidence.

Another important kind of evidence for translation is assent/dissent behaviour that indicates the logical relations of sentences. Quine uses such evidence to translate the truth functions ([8], pp. 57-61). Suppose that conjoining ' $\$$ ' with sentences $A$ and $B$ produces an acceptable sentence to which the native usually assents when, and only when, he assents to each of A and B separately. Then ' $\$$ ' should probably be translated as 'and', on the ground that 'and' plays a similar role in the translating language. Quine concedes that similar methods could be used to translate the quantifiers, though not to make the distinction between substitutional and objectual quantifiers ([9], pp. 314-15).

When Quine claims that after proxy function reassignments 'the observation sentences remain associated with the same sensory stimulations as before, and the logical interconnections remain intact', he is claiming that the proxy function and standard translations are equally acceptable on both of these sorts of evidence. But if we return to our example, I think we will see that the proxy function and standard translations are not equally supported by either kind of evidence.

As to the first sort: There are possible stimulations that would cause me to dissent from 'Mt. Everest exists', and those same stimulations, coupled with the sorts of stimulations that would typically lead me to assent to 'London is foggy', would leave me still assenting to 'London is foggy', while not assenting to ' $f$ (London) is such that its $f$-inverse is foggy'. For I would then regard ' $f$ (London)' as non-referring. In the terms of Quine's more recent account: there are perceptual situations in which I would assent to one of these translations but not the other. The two translations are not associated with the same sensory stimulations, so one may be a better match in this respect for the object language sentence. In our example the homophonic translation is better.

This objection does not deny that the defined proxy function is a proxy function. It merely points out that the translator may have good (but misleading) evidence that it isn't. The translations share truth values, but they need not, for that reason alone, be associated with the same range of sensory stimulations or perceptual situations. 


\section{STEVEN L. REYNOLDS}

Putnam's device of defining the proxy function so as to guarantee likeness of truth value in all possible worlds does not affect this point ([6], pp. 217-18). Suppose we define the proxy function as above for any possible world in which London, Mt. Everest, and the Moon all exist, and let it be the identity function for all other worlds. Then the standard translation and the proxy function translation have the same truth value in all possible worlds. They are necessarily equivalent. Suppose furthermore that I firmly believe this, as in fact I do. Still, if I received sufficient sensory evidence that Mt. Everest does not and never did exist and that London is foggy, I would then assent to 'London is foggy' but not to ' $f$ (London) is the $f$-inverse of a foggy place'. For if I ceased to believe in Mt. Everest, I would cease to believe in the necessary equivalence of the two translations.

It might be thought that Quine's argument needs only a small amendment to prevent the possibility of misleading evidence of nonexistence. The example shows however that a guarantee of likeness in truth values, even a known and properly appreciated guarantee, does not necessarily result in similar associations with sensory stimulations. If a guarantee of similar truth values does not yield similar associations with sensory stimulations, why should we expect that a guaranteed likeness in truth values plus a device to rule out evidence of non-existence will yield similar associations with sensory stimulations?

A philosopher without Quine's other commitments might answer this question by saying that, if we can argue from the truth (or falsity) of the standard translation to the truth (or falsity) of a proxy function translation using only mathematical or logical premisses, the two translations should be regarded as having the same empirical content. Mathematics adds no empirical content. In the example, the argument from the truth of the standard translation to the truth of the proxy function translation presupposes the existence of Mt. Everest. Eliminate this empirical contamination, and we should expect the translations to have the same associations to sensory stimulation, since there would be an a priori argument from one to the other.

As a matter of fact, learning mathematics does tend to alter the sensory stimulations to which we respond by assenting to other sentences. So why should we regard mathematics as lacking in empirical content, rather than, with Quine, regarding it as having empirical content in virtue of its role in (undoubtedly) empirical theories? Quine would not adopt the analytic/ synthetic distinction, in the objectionable (to him) form of lacking/having empirical content, just to save the proxy function argument.

My objection seems to depend on the epistemic possibility that a singular term in the proxy function translation fails to refer. But Quine 
eliminates singular terms from his canonical language for science, replacing them with constructions involving quantifiers, variables, and (often contrived) predicates. Can the objection be avoided by requiring that the translations be made into Quinean canonical languages?

It is easy to see that this won't do it. Under the sensory conditions in which I would not assent to sentences containing ' $f$ (London)', I would also not assent to sentences that entail that something uniquely $f$ (London)-izes. ${ }^{2}$

One might try to evade the objection by requiring that the proxy function switches of referents always take objects to objects that are unavoidably believed to be necessary existents, say, numbers. We might use a proxy function that takes London to the number 1, Mt. Everest to 2, the Moon to 3, each natural number $n$ to $n+3$, and everything else to itself. But, as we have noted, Quine does not hold that mathematics lacks empirical content: sufficiently recalcitrant sensory experience might lead us to change any of our judgments, including, perhaps, as a last resort, our mathematical judgments ([8], pp. 12-13). Exposure to philosophical argument might also undermine belief in numbers (Field, [4]). Furthermore, even supposing that the modified argument works, its conclusion must sound weak to all but the most Platonically inclined. Instead of concluding that, when I use 'London', I could be referring to anything, as in the original argument, the amended argument concludes that either I refer to London or I refer to some object whose existence no possible experience can properly cause me to doubt.

Another promising amendment is to require a conditional definition of the proxy function. So $f$ (London) is Mt. Everest, if Mt. Everest exists, but it is London otherwise. Since Mt. Everest does exist the proxy function translation will treat object language occurrences of 'London' as referring to Mt. Everest. But should the translator come to believe Mt. Everest doesn't exist, she will then think that ' $f$ (London)' refers to London, and so she will still assent to ' $f$ (London) is such that its $f$-inverse is foggy'.

Suppose, for the sake of argument, that this device or some other produces sentences that are assented to or dissented from under the same stimulations as the standard translations. Even if some proxy function translations pass this test, they will still not be equivalent to the standard translation on Quine's other kind of evidence for translation. From 'London is foggy' I am wont to infer that something is London and something is foggy. From ' $f-1$ (London) is foggy', on the other hand, I am wont

2 'A word now about reparsing singular terms as general terms. When I have urged that course for certain purposes, I have suggested that any presumption of existence and uniqueness that had been regarded as intrinsic to the singular terms might be regarded thereafter as intrinsic to the corresponding general terms.' (Quine [11], p. 114) 


\section{STEVEN L. REYNOLDS}

to infer that something is $f^{-1} f$ (London), that something is $f$ (London), that something is London, and that something is foggy. The proxy function translation is richer in available existential inferences than is the standard translation.

So it seems that, contrary to Quine's assertion, the logical interconnections do not remain intact on the proxy function translation. The proxy function translation, but not the standard translation, has logical interconnections that do not appear for the object language sentence.

It might be argued that these latter sorts of mismatch have nothing to do with reference, for they would occur even if the proxy function were defined as the identity function. ${ }^{3}$ The referents assigned would then be the same as on the standard interpretation, yet there would be mismatches in the logical relations to other sentences.

But the argument claimed that all evidence for reference is evidence for translation, and it tried to show that the rival schemes are equally well supported by the evidence for reference by showing that they are equally well supported by the evidence for translation. Although failure to match on logical interconnections doesn't show that there is something wrong with the reference scheme, it does obstruct the intended argument. Since the alternative translations are not equally supported, it does not follow that the different referential assignments are equally supported.

To make the argument work it now appears to be necessary to show how to draw a distinction between evidence for translation and evidence for reference, and to show that the logical interconnections just described are not evidence for reference (even when rival reference schemes cannot be assigned without failures in translation). But then there appears to be a conflict with Quine's often reiterated view that the sentence, and not any subsentential expression, is the smallest unit of language that has any direct connection to the evidence.

Finally it is necessary to say something about Putnam-style responses to objections to the proxy function argument. They offer a proxy function reinterpretation of the objection, or of selected predicates used in it (Putnam, [6], p. 36; [7], pp. 112-13). In giving the proxy function argument, Quine tries to persuade us to give up the traditional view of reference. To succeed he must argue from premisses acceptable to us. We do not at present regard proxy function reinterpretations as equally acceptable with the standard interpretation. So to offer to reinterpret objections with a proxy function is to abandon the attempt at persuasion.

\footnotetext{
3 This suggestion is due to Alexander George.
} 
Quine might offer such reinterpretations if he aimed only to repel arguments against the indeterminacy thesis. As persuasion to give up the traditional view of reference however, the proxy function argument fails. ${ }^{4}$

Arizona State University

Tempe, AZ 85287-2004

atslr@asuvm.inre.asu.edu

\section{References}

[1] Donald Davidson, 'The Inscrutability of Reference', in his Inquiries into Truth and Interpretation (Oxford: Clarendon Press, 1984) 227-41.

[2] Michael Devitt, Realism and Truth (Oxford: Basil Blackwell, 1984).

[3] Hartry Field, 'Conventionalism and Instrumentalism in Semantics', Nous 9 (1975) $375-405$.

[4] Hartry Field, Science Without Numbers (Princeton NJ: Princeton University Press, 1980).

[5] Robert Kirk, Translation Determined (Oxford: Clarendon Press, 1986).

[6] Hilary Putnam, Reason, Truth and History (Cambridge: Cambridge University Press, 1981).

[7] Hilary Putnam, Realism with a Human Face (Cambridge, MA: Harvard University Press, 1990).

[8] W. V. Quine, Word and Object (Cambridge MA: The M.I.T. Press, 1960).

[9] W. V. Quine, 'Replies', in Words and Objections, ed. Donald Davidson and Jaakko Hintikka (Dordrecht: D. Reidel, 1969).

[10] W. V. Quine, 'On the Very Idea of a Third Dogma', in his Theories and Things (Cambridge, MA: Harvard University Press, 1981).

[11] W. V. Quine, 'Reply to Dagfinn Føllesdal', in The Philosophy of W. V. Quine, ed. L. E. Hahn and P. A. Schilpp (La Salle: Open Court, 1986).

[12] W. V. Quine, Pursuit of Truth, Revised Edition (Cambridge, MA: Harvard University Press, 1992)

[13] John Wallace, 'Only in the Context of a Sentence Do Words Have Any Meaning', Midwest Studies in Philosophy 2, ed. P. A. French, T. E. Uehling, Jr., and H. K. Wettstein, (Morris: University of Minnesota Press, 1977) 144-64.

4 Thanks to Brad Armendt, Stewart Cohen, J. Richard Creath, Alexander George, Bernard W. Kobes, Michael J. White, Peter Smith and an anonymous referee for Analysis for helpful comments. An earlier version of this paper was presented at the American Philosophical Association meeting in December 1993. 